\title{
Genome Comparison of Actinomyces Naeslundii with known Probiotics
}

Noor Afifah Hanin Mohamad ${ }^{1}$, Mohd Hafiz Arzmi², Noratikah Othman²

${ }^{1}$ Department of Basic Medical Sciences, Kulliyyah of Nursing Internasional Islamic Univesity Malaysia, Kuantan, Pahang, Malaysia

${ }^{2}$ Department of Fundamental Dental and Medical Sciences, Kulliyyah of Dentistry, Internasional Islamic Univesity Malaysia, Kuantan, Pahang, Malaysia2

Introduction: Actinomyces naeslundii is an oral microbiome that has been suggested to involve in actinomycosis particularly in immunocompromised patients. However, studies have also shown that the bacterium can inhibit the colonisation of Candida albicans and cariogenic bacterium, Streptococcus mutans. Even though various studies have been conducted to characterise A. naeslundii, however, the role of the bacterium in the oral cavity either to induce pathogenicity or to improve oral health remain unclear. The objective of this study is to determine the role of A. naeslundii in the oral cavity using bioinformatic tools with the hypothesis that A. naeslundii possesses genome similarity to oral probiotics. Materials and method: The study was conducted by data mining of the genomic sequences of A. naeslundii NCTC10301 from GenBank. Following that, genomic comparison was performed with 25 species of well-known probiotics. MAUVE version 2.3.1 was used to find similarities between A. naeslundii with the known probiotics through identification of potential relevance probiotic properties in A. naeslundii. Phylogenetic tree was constructed using PHYLIP package to identify evolutionary relationship of $A$. naeslundii with the known probiotics. The $16 \mathrm{~s}$ rRNA sequences of $A$. naeslundii and known probiotics were harvested from GenBank using BLAST. Finally, the functions of all A. naeslundii genes that shared similarity with known probiotics were identified using INTERPRO. Result: Genome comparison analysis of the present study showed that A. naeslundii genome exhibited high similarity to Bifidobacterium species such as $B$. animalis subsp. lactis, B. bifidum, B. longum and B. breve. In addition, phylogenetic tree analysis showed that Bacillus species, B. subtilis and B. cereus, appeared to clade together with $A$. naeslundii, with bootstrap value of $98 \%$. Conclusion: A. naeslundii had high similarity with probiotic Bifidobacterium species thus supported the hypothesis of the present study that $A$. naeslundii possesses genome similarity to oral probiotic. 